where the hypertensive response to isometric hand grip exercise is discussed, it would have been nice to see a reference to Lind and his colleagues who first demonstrated the response.

It was good to see a chapter about autonomic failure in the elderly. The extensive studies of the incidence of orthostatic hypotension, thermoregulatory impairment and the relationship of Parkinsons and cerebrovascular diseases to autonomic dysfunction in older people are very well discussed.

Almost every form of autonomic dysfunction from multiple system atrophy, familial dysautonomia, amyloid disruption of autonomic fibres, to problems of autonomic outflow to the eyes and many others are well discussed, with descriptions of clinical presentations, pathology, neurochemistry and investigative procedures. Associated problems such as ion and fluid homeostasis alterations receive attention.

The book ends with an exciting chapter on experimental studies of immune autonomic neuropathies. Throughout the book, present knowledge is supplemented with exciting experimental work in progress, and quantitative measuring techniques are presented to aid our studies of patients with autonomic nervous system disease, and attention is given to the difficult problems of management of these patients. The reference lists are extensive and the book is well indexed. It is a "state of the art" book, which, by its many stimulating discussions, will promote research work which will produce a need for a new edition in a few years. Though fairly expensive, this book is mandatory for the bookshelves of any physician, neurosurgeon or physiologist with a major interest in the autonomic nervous system.

\section{K.E. Cooper} Calgary, Alberta

TOPICS IN NEONATAL NEUROLOGY. 1984. Published by Grune and Stratton. Edited by H.B. Sarnat. 301 pages.

Neonatal neurology, as a sub-discipline of neurology, has developed extremely rapidly during the past decade. A veritable explosion of new knowledge has occurred, both from the basic science and clinical points of view, largely prompted by technological advances which have enhanced the survival of critically-ill neonates. Several textbooks on neonatal neurology, some attempting to be encyclopedic, have appeared in the last few years. Unfortunately the exceptionally rapid evolution of knowledge about the developing brain and its many disorders has led to early obsolescence of some of the information in these books.

The recently published book, "Topics in Neonatal Neurology", has not been designed as another exhaustive review, but as a selective reassessment of several major problems in neonatal neurology with a view to updating knowledge in these areas and, hopefully, provoking further discussion and inquiry. Given these restricted goals, the authors of "Topics in Neonatal Neurology" have succeeded quite well in their endeavours.

Among the authors of this volume are a number of researchers who have made important contributions to the science of the neonatal brain and who remain in the forefront of their respective fields. Topics covered in the book include perinatal cerebrat hypoxia-ischemia, periventricular and intraventricular hemorrhage, bilirubin encephalopathy, hyperammonemic encephalopathy, neonatal meningitis, apnea, and seizures. In addition there is a timely review of neonatal ultrasound, EEG and evoked potentials. The volume is prefaced by an interesting correlation of the anatomophysiologic changes in the developing brain and evolving behavioural phenomena in the premature infant.

On the whole the chapters are well-written and concise. There is a clear attempt to make the text easily comprehensible to readers from related fields. Relevant animal experimental data is presented carefully without losing the reader in a welter of conflicting information; the emphasis in each chapter remains that of the fundamental clinical problem for the practising physician. Reference material is up-to-date and appropriate without being exhaustive. There are unfortunately a disconcerting number of typographical errors in the text, sometimes of fairly major importance (eg. pictures incorrectly labelled or in reverse order to that described in the text). With this exception, "Topics in Neonatal Neurology" is a well-conceived book which will be of use, in particular, to pediatric neurologists, neonatologists and pediatricians involved in the care of sick newborns.

P. Humphreys Ottawa, Ontario

ENTRAPMENT NEUROPATHIES. 1983. By D.M. Dawson, M. Hallett, L.H. Millender. Published by Little, Brown \& Co.

Entrapment neuropathies are very common and their elucidation is important to a wide spectrum of general practitioners and specialists. In the last two decades, much has been learned of the pathophysiology of these entities. Electrophysiological techniques have now evolved to the point that localization and the probable underlying character of an entrapment neuropathy can be determined reliably.

This book is a collaborative effort of two neurologists and an orthopedic surgeon. It provides discussion of clinical presentations, differential diagnoses, electrophysiological diagnostic techniques and management placing particular emphasis on the more common neuropathies. The bibliography is both current and comprehensive and the illustrations helpful (although more intraoperative photographs are included than seems necessary to make the essential points).

This new volume is recommended as a concise yet adequately comprehensive guide to the recognition and management of entrapment neuropathies. It is well worth its price.

\section{W.F. Brown, P. Barton London, Ontario}

PATHOLOGY OF SKELETAL MUSCLE. By Sterling Carpenter and George Karpati. Published by Churchill Livingstone. 754 pages. $\$ 132.50 \mathrm{Cdn}$.

In the first sentence of the preface, the authors stated that "the central purpose of this book is to provide an accurate description of the pathology of skeletal muscle". I believe that they have succeeded admirably in doing this.

Approximately half of the book is devoted to a description of:

a) the general pathologic reactions that affect muscle fibers,

b) the normal organelles and constituents of muscle fibers and how they react pathologically,

c) abnormal structures that can be found in muscle fibers, and 